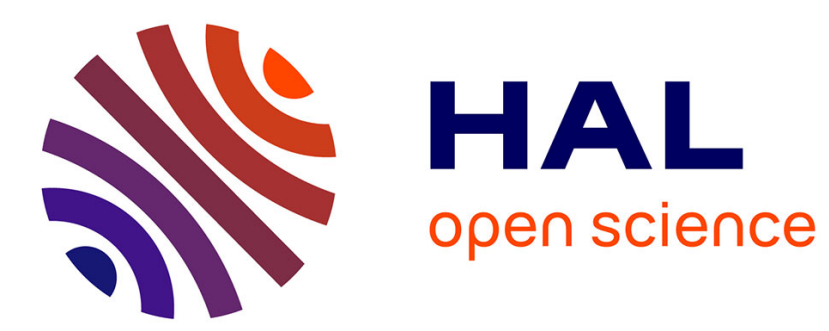

\title{
A numerical investigation of the overlap distribution among pure states in the spin glass phase
}

\author{
N. Parga, G. Parisi, M.A. Virasoro
}

\section{To cite this version:}

N. Parga, G. Parisi, M.A. Virasoro. A numerical investigation of the overlap distribution among pure states in the spin glass phase. Journal de Physique Lettres, 1984, 45 (22), pp.1063-1069. 10.1051/jphyslet:0198400450220106300 . jpa-00232450

\section{HAL Id: jpa-00232450 https://hal.science/jpa-00232450}

Submitted on 1 Jan 1984

HAL is a multi-disciplinary open access archive for the deposit and dissemination of scientific research documents, whether they are published or not. The documents may come from teaching and research institutions in France or abroad, or from public or private research centers.
L'archive ouverte pluridisciplinaire HAL, est destinée au dépôt et à la diffusion de documents scientifiques de niveau recherche, publiés ou non, émanant des établissements d'enseignement et de recherche français ou étrangers, des laboratoires publics ou privés. 


\title{
LE JOURNAL DE PHYSIQUE-LETTRES
}

J. Physique Lett. 45 (1984) L-1063 - L-1069

15 NOVEMBRE 1984, PAGE L-1063

Classification

Physics Abstracts

$75.50 \mathrm{~K}$

\section{A numerical investigation of the overlap distribution among pure states in the spin glass phase}

\author{
N. Parga $\left(^{+}\right)$ \\ INFN Sezione di Roma, Italy \\ G. Parisi \\ Dipartimento di Fisica, Università Di Roma II «Tor Vergata » \\ and Laboratorio Nazionale INFN de Frascati, Italy
}

and M. A. Virasoro

Dipartimento di Fisica, Università di Roma I « La Sapienza » and INFN, Sezione di Roma, Italy

(Reçu le 10 août 1984, accepté le 27 septembre 1984)

\begin{abstract}
Résumé. - A partir des équations de champ moyen à température nulle on étudie numériquement les propriétés du modèle de Sherrington-Kirkpatrick. En particulier on observe les fluctuations du paramètre d'ordre $q(x)$ quand on change de couplages $J_{i j}$ et on voit partiellement la structure ultramétrique parmi les états purs.

Abstract. - We use zero temperature mean field equations to study numerically several properties of the Sherrington-Kirkpatrick model. In particular we observe the fluctuations from sample to sample of the order parameter $q(x)$ and see partial evidence of the ultrametricity structure among pure states.
\end{abstract}

The Sherrington-Kirkpatrick model $[1,2]$ is defined by the Hamiltonian :

$$
H=\sum_{i>j} J_{i j} s_{i} s_{j}
$$

$\left(^{+}\right)$Permanent Address : Centro Atomico Bariloche. 8400 Bariloche, Argentına. 
where $s_{i}$ are Ising variables, $i$ and $j$ run from 1 to $N$ and the $J_{i j}$ are random variables distributed according to :

$$
P\left(J_{l j}\right)=(N / 2 \pi)^{1 / 2} \exp \left(-N / 2 J_{l j}^{2}\right) .
$$

For every observable we can define :

a thermal average

$$
\begin{aligned}
& \langle\mathcal{O}\rangle=\frac{\sum_{\left\{s_{l}\right\}} \mathrm{e}^{-\beta H\left[s_{i}\right]} \mathcal{O}}{Z} \\
& \overline{\langle\mathcal{O}\rangle}=\int P\left(J_{i j}\right)\langle\mathcal{O}\rangle \prod_{i, j} \mathrm{~d} J_{l j} .
\end{aligned}
$$

Evidence has been accumulating to prove that averages $\overline{\langle\mathcal{O}\rangle}$ can be correctly calculated in the Replica Symmetry Breaking Scheme introduced in [3]. This evidence comes in part from cross investigation of the model using Mean-Field Equations (TAP equations [4, 7]), MonteCarlo simulations [5] and Small System analysis [6].

In addition these studies have led to a picture of the spin glass phase as characterized by the existence of a large number (infinite when $N \rightarrow \infty$ ) of equilibrium states almost degenerate in free energy (i.e. $E_{\alpha}-E_{\beta}=0(1)$ for $N \rightarrow \infty$ ) separated by barriers that become infinitely high in the thermodynamic limit.

A recent interpretation [8] of the order parameter $q(x)$ allows the use of the Replica Symmetry Breaking to calculate quantities that relate to this picture. In reference [8] the following definitions were introduced : the weight of the states

$$
P_{\alpha}=\exp \left(-\beta E_{\alpha}\right) / \sum_{\alpha^{\prime}} \exp \left(-\beta E_{\alpha^{\prime}}\right)
$$

the overlap between states

$$
q_{\alpha \beta}=\sum_{l} m_{i}^{\alpha} m_{i}^{\beta} / N
$$

where $m_{i}^{\alpha}$ is the local magnetization in the state $\alpha$ and the probability that two states $\alpha, \beta$ have overlap $q$

$$
P_{J}(q)=\sum_{\alpha, \beta} P_{\alpha} P_{\beta} \delta\left(q_{\alpha \beta}-q\right)
$$

It was then proved that :

$$
P(q)=\overline{P_{J}(q)}=\mathrm{d} x / \mathrm{d} q
$$

where $q(x)$ is Parisi's order parameter.

In a recent paper this analysis was further developed. Two interesting results emerged [9].

The first one is that $P_{J}(q)$ depends on the particular realization of the couplings $J_{i j}$ (see also Ref. [10]). In other words it fluctuates from sample to sample. Defining :

$$
Y_{J}(q)=\int_{q}^{q_{\max }} P_{J}\left(q^{\prime}\right) \mathrm{d} q^{\prime}
$$

and

$$
y(q)=\overline{Y_{J}(q)},
$$

the probability distribution $\Pi_{V}(Y)$ of $Y_{J}(q)$ could be numerically reconstructed from the moments. Surprisingly enough it depends explicitly only on $y$. 
The second feature is that the states $\alpha$ are distributed in phase space in such a way that the distance among them have an ultrametric organization. By definition this means that out of three states chosen at random, the two of them that are nearer to each other lie at the same distance of the third. It is important to check these properties by other methods because, in particular, the 2 nd property seems to be closely related to properties of the original ansatz used in reference [3] to break the Replica Symmetry. It has in fact been conjectured that ultrametricity is a sufficient condition to uniquely derive that form of symmetry breaking [11]. The natural way to look at this structure is to study the solutions to TAP equations $[4,5,15]$. Unfortunately these equations are very hard to analyse numerically because convergence to a solution rarely occurs. On the other hand it is reasonable to think that properties 1) and 2) do not depend on details about the dynamics but are rather common to any model with a spin glass phase. Inspired by a recent paper [12] and the PAT scaling [13] at $T=0$ we propose the following " model». We look at solutions of $T=0$ mean field equations :

$$
m_{i}=\operatorname{sign}\left(\sum_{i} J_{i j} m_{j}\right), \quad m_{i}= \pm 1
$$

and use as Boltzmann-Gibbs weights :

$$
P=\exp \left(-\delta \sum_{i, j} m_{i} J_{i j} m_{j}\right) .
$$

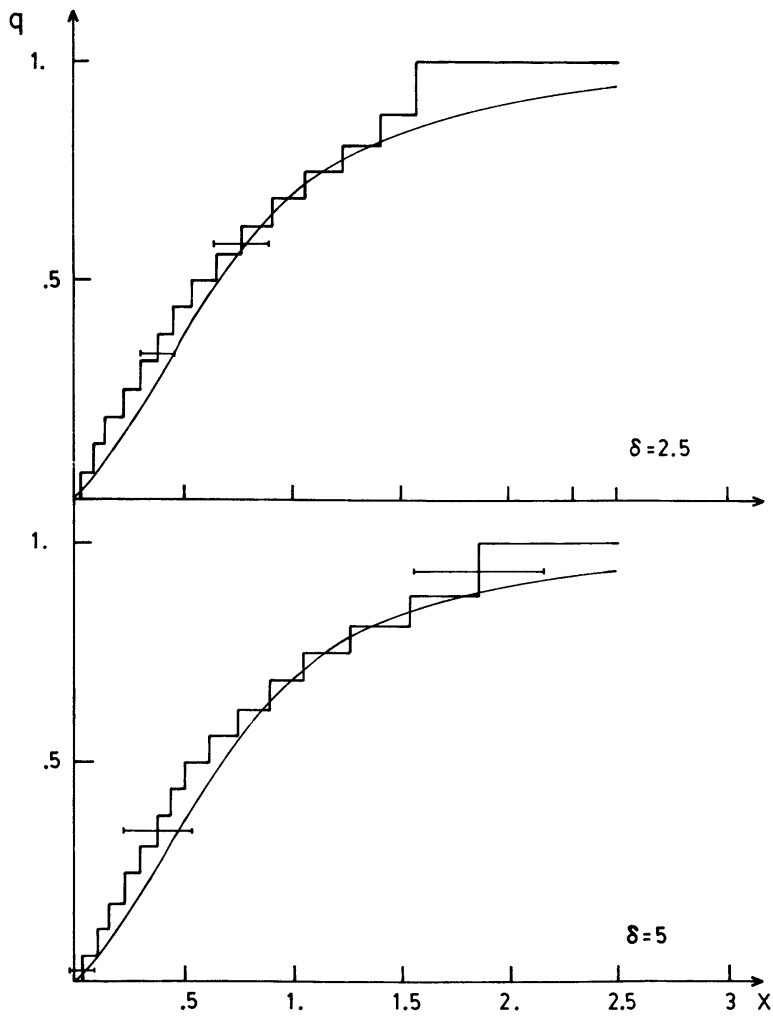

Fig. 1. - The order parameter $q(x)$ for $N=32$ and two different values of $\delta$. The curve corresponds to the predictions of Ref. [13]. For a finite system only a finite number of values of the overlap are possible, hence the steps in our results. 
Then by using results of both references we expect on one hand that

$$
q_{\mathrm{SK}}(x, \beta) \simeq \tilde{q}(\beta x) \text { for } \beta \rightarrow \infty \text { and } x<\tilde{x}(\beta)
$$

where $q_{\mathrm{SK}}(x, \beta)$ is the Parisi's function for the SK model, $\tilde{q}$ is the scaling PAT function and $\tilde{x}(\beta)$ is the end point of the plateau while changing the weight factors

$$
\exp \left(-\beta F_{\alpha}\right) \rightarrow \exp \left(-\beta u F_{\alpha}\right)
$$

should produce a model with an order parameter $q_{u}(x)$ such that

$$
q_{u}(x, \beta)=q_{\mathrm{SK}}(|u| x, \beta) .
$$

Therefore in our case we anticipate a $q(x)$ of the form

$$
q(x) \begin{cases}=\tilde{q}(\beta|u| x)=\tilde{q}(\delta x) & \text { in the range } \delta x<\tilde{x}(\infty) \\ =1 & \text { in the range } \delta x>x(\infty) .\end{cases}
$$

Notice that by changing $\delta$ we can check this last equation. In order to obtain $q(x)$ we solve equation (11) finding all solutions which are relevant for the particular value of $\delta$ (in practice we cut solutions with a relative weight with respect to the ground state smaller than $10^{-4}$ ). The algorithm we use to find the lower lying solutions consists in starting always from random uncorrelated configurations in such a way that the search for new solutions is not biased [14]. After calculating

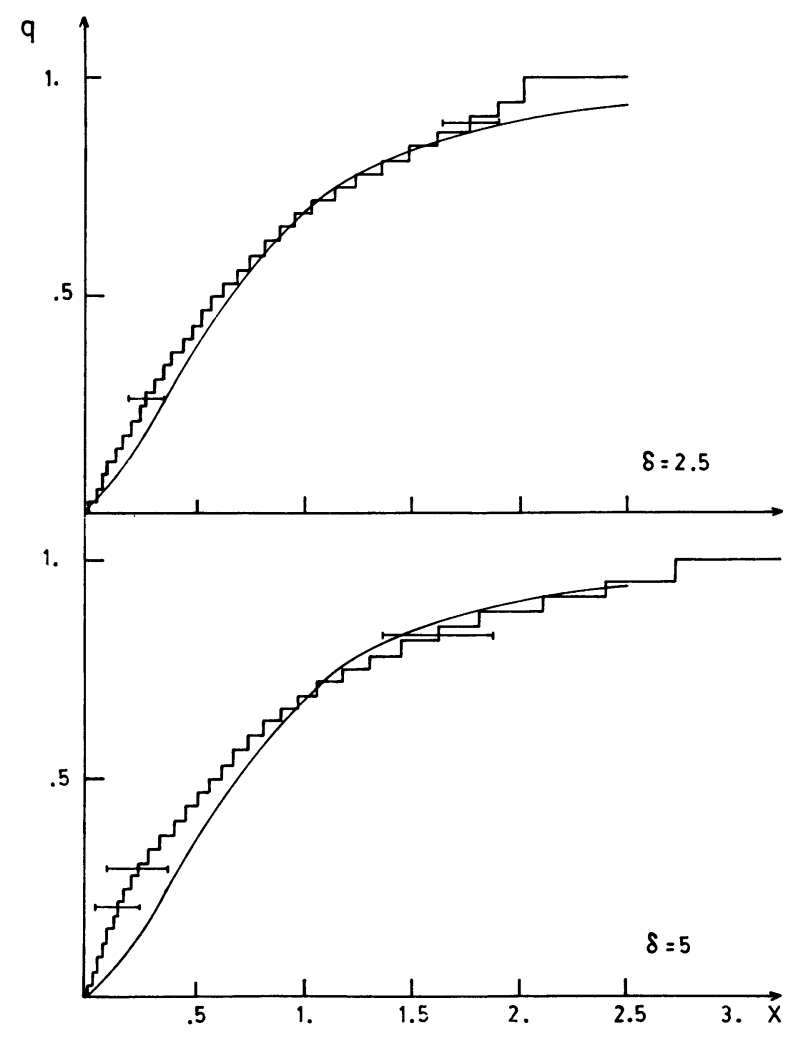

Fig. 2. - Idem as in figure 1 but for $N=64$. 
the corresponding overlaps we evaluated $P_{J}(q)$ and by integration $q_{J}(x)$. To obtain $q(x)$ we took the average over 150 different coupling configurations. The results obtained for $N=32$ and $N=64$ and different values of $\delta$ are shown in figure 1 and 2 where it is also exhibited the function $q(x)$ obtained in reference [14] for the infinite system. Once we have the $P_{J}(q)$ for a large number of coupling configurations it is straightforward to obtain its probability law $\Pi_{y}(Y)$. From our 150 configurations we obtained results in qualitative agreement with those of reference [9]. As an example we give in figure 3 the probability distribution of $Y$ when $y$ equals 0.69 and 0.63 for two different values of $\delta$. We see that both curves are in a good agreement with $\Pi_{y}(Y)$ being a function of $y$ only; the values of the overlaps are of course different although related by the rescaling $v_{\delta_{1}}\left(q_{1}\right)=y_{\delta_{2}}\left(q_{2}\right)$.

We now consider the probability distribution of three overlaps

$$
P_{J}\left(q_{1}, q_{2}, q_{3}\right)=\sum_{\alpha \beta \gamma} P_{\alpha} P_{\beta} P_{\gamma}\left(q_{1}-q_{\alpha \beta}\right)\left(q_{2}-q_{\alpha \gamma}\right)\left(q_{3}-q_{\beta \gamma}\right)
$$

which, as shown in reference [9], after averaging over the coupling configurations exhibits the ultrametric topology of the space of pure states :

$$
\begin{aligned}
P\left(q_{1}, q_{2}, q_{3}\right)=0.5 P\left(q_{1}\right) \times\left(q_{1}\right) \delta\left(q_{1}-q_{2}\right) \delta\left(q_{2}-q_{3}\right)+ & \\
& +0.5\left\{P\left(q_{1}\right) P\left(q_{2}\right) \delta\left(q_{1}-q_{2}\right) \delta\left(q_{2}-q_{3}\right)+\text { perm }\right\} .
\end{aligned}
$$

We evaluated the following moments using our solutions of the TAP equations :

$$
\begin{aligned}
& M_{12}\left(q_{3}\right)=\int_{q_{1} \leqslant q_{2} \leqslant q_{3}} P\left(q_{1}, q_{2}, q_{3}\right)\left(q_{1}-q_{2}\right)^{2} \mathrm{~d} q_{1} \mathrm{~d} q_{2} \\
& M_{23}\left(q_{3}\right)=\int_{q_{1} \leqslant q_{2} \leqslant q_{3}} P\left(q_{1}, q_{2}, q_{3}\right)\left(q_{2}-q_{3}\right)^{2} \mathrm{~d} q_{1} \mathrm{~d} q_{2} \\
& M_{13}\left(q_{3}\right)=\int_{q_{1} \leqslant q_{2} \leqslant q_{3}} P\left(q_{1}, q_{2}, q_{3}\right)\left(q_{1}-q_{3}\right)^{2} \mathrm{~d} q_{1} \mathrm{~d} q_{2} .
\end{aligned}
$$

According to equation (18) the first of these moments should tend to zero in the thermodynamic limit while the other two should become equal. We evaluated the three moments for different

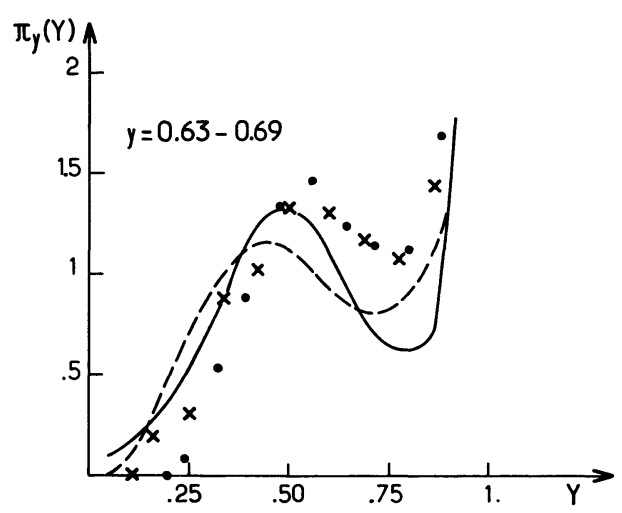

Fig. 3. - The probability distribution of $Y_{J}$ for $\delta=2.5$ (dots) and for $\delta=5$ (crosses). $N=32$. The curves are reproduced from Ref. [9] and correspond to two different reconstruction of the probability distribution from the moments. We have added data with $y=0.69$ and $y=0.63$. 
values of $q_{3}$ and for $N=32$ and $N=64$. We can see in table I that $M_{12}$ seems to converge more rapidly to its limiting value than the other two. This is reasonable because any anomaly will contribute quadratically to $M_{12}$ and linearly to $M_{23}$ and $M_{13}$.

Table I. - Moments of the probability distribution of three overlaps as defined in Eq. (19). The value of $\delta=10$.

\begin{tabular}{|c|c|c|c|c|c|c|}
\hline \multirow{2}{*}{$q_{3}$} & \multicolumn{2}{|c|}{$M_{12}\left(q_{3}\right)$} & \multicolumn{2}{|c|}{$M_{32}\left(q_{3}\right)$} & \multicolumn{2}{|c|}{$M_{13}\left(q_{3}\right)$} \\
\hline & $N=32$ & $N=64$ & $N=32$ & $N=64$ & $N=32$ & $N=64$ \\
\hline 0.5 & $1.79 .10^{-5}$ & $3.51 .10^{-6}$ & $7.29 \cdot 10^{-5}$ & $1.55 .10^{-5}$ & $3.28 \cdot 10^{-5}$ & $6.11 .10^{-6}$ \\
\hline 0.625 & $1.37 .10^{-5}$ & $2.46 \cdot 10^{-6}$ & $5.01 \cdot 10^{-5}$ & $1.78 \cdot 10^{-5}$ & $1.72 .10^{-5}$ & $9.48 \cdot 10^{-6}$ \\
\hline 0.75 & $3.69 .10^{-5}$ & $3.76 .10^{-6}$ & $1.80 \cdot 10^{-4}$ & $8.86 \cdot 10^{-5}$ & $1.01 .10^{-4}$ & $7.41 \cdot 10^{-5}$ \\
\hline 0.875 & $2.27 .10^{-5}$ & $1.68 \cdot 10^{-5}$ & $9.46 \cdot 10^{-4}$ & $2.39 \cdot 10^{-4}$ & $8.64 \cdot 10^{-4}$ & $1.77 .10^{-4}$ \\
\hline
\end{tabular}

We can therefore conclude that $N=64$ is " more " ultrametric than $N=32$. It is much more difficult to say whether we have really observed ultrametricity. Even with 150 coupling configurations (which with our cut in energy means 59486 triangles of states for $N=64$ and 277286 triangles for $N=32$ ) the probability distribution of three overlaps turns out to have large statistical errors. In addition it is clear that the corrections in $1 / N$ are very subtle. For a decisive test we must first assure that none of the sides of the triangle have zero length when $N \rightarrow \infty$, while checking at the same time that the difference between the two larger sides does tend to zero. Furthermore we have to compare our data with a " no structure " hypothesis. We chose then the larger overlap to be $2[N / 3]$, fix one of the other sides to be a value between $2[N / 6]$ and $2[N / 3]$ and compute :

$$
U=\frac{\int_{0}^{q_{2}} \mathrm{~d} q_{1} P\left(q_{1}, q_{2}, q_{3}\right)\left(q_{1}-q_{2}\right)^{2}}{\int_{0}^{q_{2}} \mathrm{~d} q_{1} P\left(q_{1}, q_{2}, q_{3}\right)} * \frac{\int_{0}^{q_{2}} \mathrm{~d} q_{1}}{\int_{0}^{q_{2}} \mathrm{~d} q_{1}\left(q_{1}-q_{2}\right)^{2}}
$$

Table II. - Value of $U$ as defined in Eq. (20).

\begin{tabular}{|l|l|l|}
\hline \multirow{2}{*}{$q_{2}$} & \multicolumn{2}{|c|}{$U$} \\
\cline { 2 - 3 } & $N=32$ & $N=64$ \\
\hline 0.3125 & 1.09 & 1.06 \\
0.375 & 0.87 & 0.71 \\
0.4375 & 0.47 & 0.24 \\
0.500 & 0.33 & 0.23 \\
0.5625 & 0.34 & 0.093 \\
0.625 & 0.23 & 0.068 \\
\hline
\end{tabular}


The results are summarized in table II. Our data fits better the ultrametricity hypothesis than the « null » hypothesis, i.e. the values of $U$ are in general nearer to zero than to one. However we also observe that, as expected, the limit is far from uniform. We conclude that results from $N=32$ and $N=64$ spins are consistent with ultrametricity but that we need to go to larger $N$ if we want really to prove it.

\section{Acknowledgments.}

We acknowledge interesting conversations on the problem of testing the ultrametric property with M. Mezard, N. Sourlas and G. Toulouse.

\section{References}

[1] Sherrington, D. and KirkPatrick, S., Phys. Rev. Lett. 32 (1975) 1792.

[2] Edwards, S. F. and Anderson, P. W., J. Phys. F 5 (1975) 965.

[3] Parisi, G., Phys. Rev. Lett. 43 (1979) 1754 ; J. Phys. A 13 (1980) L-115, 1101, 1887.

[4] Thouless, D. J., Anderson, P. W. and Palmer, R., Philos. Mag. 35 (1977) 593.

[5] Mackenzie, N. and Young, A. P., Phys. Rev. Lett. 49 (1982) 301. Young, A. P., Phys. Rev. Lett. 51 (1983) 1206.

[6] Young, A. P. and KirkPatrick. S.. Phvs. Rev. B 25 (1982) 440.

[7] Bray, A. J. and Moore, M. A., J. Phys. C 13 (1980) L-469:

De Dominicis, C., Gabay, M., Garel, T., Orland, H., J. Physique 41 (1980) 923.

[8] Parisi, G., Phys. Rev. Lett. 50 (1983) 1946.

[9] Mezard, M., Parisi, G., Sourlas, N., Toulouse, G. and Virasoro, M., Phys. Rev. Lett. 52 (1984) 1156 ; J. Physique 45 (1984) 843.

[10] Young, A. P., Bray, A. J. and Moore, M. A., J. Phys. C 17 (1984) L-155.

[11] MeZARD, M., private communication.

[12] Bray, A. J., MoOre, M. A. and Young, A. P., preprint M/C TH-83/28.

[13] Vannimenus, J., Toulouse, G. and Parisi, G., J. Physique 42 (1981) 565.

[14] Palmer, R. G. and Pond, C. M., J. Phys. F9 (1979) 1451.

[15] De Dominicis, C. and Young, A. P., J. Phys. A 16 (1983) 2063. 